whether we can take $V=\Gamma$, when $\Gamma$ is $K$-projective. If this is found possible, we shall have shown that the relative cohomology does reduce to the absolute cohomology when we take $\Gamma=K$.

\title{
REFERENCES
}

1. H. Cartan and S. Eilenberg, Homological algebra, Princeton Univ. Press, Princeton, N. J., 1956.

2. G. Hochschild, Relative homological algebra, Trans. Amer. Math. Soc. 82 (1956), 246-269.

3. U. Shukla, Cohomologie des algèbres associatives, Ann. Sci. Ecole Norm. Sup. 78 (1961), 163-209.

Kurukshetra University, Kurukshetra (Punjab), India

\section{DIRECT PROOF OF THE BASIC THEOREM ON MULTIPARTITE PARTITIONS}

E. M. WRIGHT ${ }^{1}$

In what follows all small latin letters denote non-negative integers, while $N$ and $N_{m s}$ are $j$-partite numbers, i.e. vectors or row-matrices of $j$ dimensions whose components are non-negative integers. In particular, $N=\left(n_{1}, n_{2}, \cdots, n_{j}\right)$. We write $q_{k}(N)$ for the number of partitions of $N$ into just $k$ parts and $r_{k}(N)$ for the number of partitions of $N$ into just $k$ different parts.

Let $\pi=\pi(k)$ be a partition of $k$ into $h(1)$ parts $1, h(2)$ parts 2 and so on, so that $k=\sum_{m} m h(m)$. We write

$$
H(\pi)=\prod_{m}\left\{h(m) ! m^{h(m)}\right\}^{-1}
$$

where, as usual, $0 !=1$, and $D(\pi, N)$ for the number of solutions of

$$
N=\sum_{m} \sum_{s=1}^{h(m)} m N_{m s},
$$

where the order of the $N_{m s}$ is relevant. Clearly

$$
D(\pi, N)=\prod_{i=1}^{j} D\left(\pi, n_{i}\right)
$$

Again, for $|X|<1$,

Received by the editors February 3, 1963.

1 Research sponsored by the Office, Chief of Research and Development, U. S. Department of the Army, through its European Research Office under contract DA91-591-EUC-2670. 


$$
1+\sum_{n=1}^{\infty} D(\pi, n) X^{n}=\prod_{m}\left(1-X^{m}\right)^{-h(m)} .
$$

Hence, for any given $\pi, D(\pi, n)$ can be readily evaluated.

These last facts give their value to the two results

$$
q_{k}(N)=\sum_{\pi(k)} H(\pi) D(\pi, N)
$$

and

$$
r_{k}(N)=\sum_{\pi(k)}(-1)^{k-\Sigma h(m)} H(\pi) D(\pi, N) .
$$

In [1] I found relations between generating functions equivalent to these and I exploited them to calculate $q_{k}(N)$ and $r_{k}(N)$ for small $k$ in [2]. The equalities (3) and (4) are, however, clearly enumerative relations which have nothing essentially to do with $j$-partite numbers and which should be capable of direct enumerative proof. Such a proof I give here. While I could wish it simpler, it does give some insight into the nature and significance of (3) and (4).

We write

$$
\rho(k)=\sum_{\pi(k)} H(\pi), \quad \mu(k)=\sum_{\pi(k)}(-1)^{k-\Sigma h(m)} H(\pi)
$$

and prove first that

$$
\begin{gathered}
\rho(k)=1, \\
\mu(1)=1, \quad \mu(k)=0 \quad(k>1) .
\end{gathered}
$$

These are trivial, if we use generating functions. We have only to expand both sides of $(1-X)^{-1}=\exp \{-\log (1-X)\}$ and of $1+X$ $=\exp \log (1+X)$ in ascending powers of $X$ and equate coefficients of $X^{k}$. But our present aim excludes this approach. The following proofs of (5) and (6) avoid the use of generating functions.

We use the elementary theory of the symmetric group of permutations on $k$ elements. The order of this group is $k$ ! and it can be split up into classes of conjugate elements, each class corresponding to a partition of $k$. The class corresponding to the partition $\pi$ of $k$ (i.e. all those permutations which can be expressed by $h(m)$ cycles of length $m$, etc.) has

$$
k ! \prod_{m}\left\{h(m) ! m^{h(m)}\right\}^{-1}=k ! H(\pi)
$$

members. Hence

$$
k !=\sum_{\pi(k)} k ! H(\pi)
$$


Dividing both sides by $k$ !, we have (5).

A permutation which consists of a single cycle of length $m$ is positive or negative according as $m-1$ is even or odd. Hence every permutation of the class corresponding to $k$ is positive or negative according as $k-\sum h_{m}$ is even or odd. For $k>1$, there are as many positive as negative permutations and so

$$
k ! \mu(k)=\sum_{\pi(k)}(-1)^{k-\Sigma h(m)} k ! H(\pi)=0,
$$

but $\mu(1)=1$ obviously. Hence (6).

Let us suppose that $h(m, t)$ of the $N_{m s}$ on the right of (1) are equal to $N_{t}$ so that

$$
N=\sum_{t=1}^{r} k_{t} N_{t}
$$

where

$$
k_{t}=\sum_{m} m h(m, t)
$$

and all the $N_{t}$ are different. Let $\pi_{t}=\pi_{t}\left(k_{t}\right)$ be the partition of $k_{t}$ which has $h(m, t)$ parts equal to $m$. Then $\pi$ separates into the partitions

$$
\pi_{1}+\pi_{2}+\cdots+\pi_{r}
$$

If, for a fixed $m$, we permute the $N_{m s}$ among themselves, we have $h(m) ! /\{h(m, 1) ! \cdots h(m, r) !\}$ different arrangements of the $N_{m s}$. Hence the same $k_{1}, \cdots, k_{r}, N_{1}, \cdots, N_{r}, \pi_{1}, \cdots, \pi_{r}$, give rise to

$$
\lambda\left(\pi_{1}, \cdots, \pi_{r}\right)=\prod_{m} h(m) ! /\{h(m, 1) ! \cdots h(m, r) !\}
$$

different solutions of (1). We remark also that

$$
H(\pi) \lambda\left(\pi_{1}, \cdots, \pi_{r}\right)=\prod_{t=1}^{r} H\left(\pi_{t}\right) .
$$

Hence, corresponding to any one partition of $N$ into $k$ parts given by (7), we have 1 on the left-hand side of (3) and on the right-hand side

$$
\begin{aligned}
\sum_{\pi(k)} H(\pi) \lambda\left(\pi_{1}, \cdots, \pi_{r}\right) & =\sum_{\pi(k)} \prod_{t=1}^{r} H\left(\pi_{t}\right) \\
& =\prod_{t=1}^{r} \sum_{\pi_{t}\left(k_{t}\right)} H\left(\pi_{t}\right)=\prod_{t=1}^{r} \rho\left(k_{t}\right)
\end{aligned}
$$

and this is 1 by (5). 
Similarly the contribution to the right-hand side of (4) is

$$
\begin{aligned}
\sum_{\pi(k)}(-1)^{k-\Sigma h(m)} H(\pi) \lambda\left(\pi_{1}, \cdots, \pi_{r}\right) & =\sum_{\pi(k)} \prod_{t=1}^{r}(-1)^{k_{t}-\Sigma h(m, t)} H\left(\pi_{t}\right) \\
& =\prod_{t=1}^{r} \sum_{\pi_{t}\left(k_{t}\right)}(-1)^{k_{t}-\Sigma h(m, t)} H\left(\pi_{t}\right) \\
& =\prod_{t=1}^{r} \mu\left(k_{t}\right)
\end{aligned}
$$

and this is 1 or 0 according as $\prod_{i=1}^{r} k_{t}$ is 1 or greater than 1, i.e. according as all the $N_{m s}$ are different or not. This is precisely the condition for this partition to contribute 1 or 0 to the left-hand side of (4).

\section{REFERENCES}

1. E. M. Wright, Partitions of multi-partite numbers, Proc. Amer. Math. Soc. 7 (1956), 880-890, especially 883 , formulae (6) and (7).

2. - Partition of multi-partite numbers into a fixed number of parts, Proc. London Math. Soc. (3) 11 (1961), 499-510.

University of Aberdeen, Scotland 